\title{
FIRST RESULTS OF A NEAR IR MONITORING PROGRAM OF OH/IR STARS
}

\author{
P. GARCíA-LARIO \\ LAEFF - Estación de Villafranca del Castillo. Apdo. 50727. E-28080 Madrid (Spain)
}

D. ENGELS

Hamburger Sternwarte. Gojenbersweg 112. D-2050 Hamburg 80 (Germany)

and

A. MANCHADO

Instituto de Astrofísica de Canarias. E-38200, La Laguna, Tenerife (Spain)

\begin{abstract}
We present the first results of a long-term monitoring program of observations in the near infrared of a selected sample of $\mathrm{OH} / \mathrm{IR}$ stars included in the IRAS Point Source Catalogue. The observations have been made using the $1.5 \mathrm{~m}$ Sánchez Magro Telescope (SMT) at Izaña (Tenerife, Spain) since the beginning of 1991 and are still in progress. They are being complemented with observations made using the $1 \mathrm{~m}$ ESO photometric telescope (La Silla, Chile). The sample includes $30 \mathrm{OH} / \mathrm{IR}$ stars with a variety of infrared and $\mathrm{OH}$ maser luminosities, expansion velocities, LRS classes and position in the IRAS two-colour diagram.

During the first phase of these observations we have identified for the first time their near infrared counterparts. In some cases we have obtained a first estimation of the amplitude $\Delta m$ and period $P$ of their photometric variations. As an example, we present the light curves of IRAS $16037+4218(\Delta m=0.75 \mathrm{mag} ; \mathrm{P}=369$ days $)$ and IRAS $06297+4045(\Delta m=1.29 \mathrm{mag} ; \mathrm{P}=697$ days $)$. Our data confirm the strong variability of these objects, which in some cases reaches more than 2 magnitudes. The near infrared colours observed in the $J-H$ vs $H-K$ colour-colour diagram can be explained as dust emission corresponding to temperatures ranging from $700 \mathrm{~K}$ to more than $1500 \mathrm{~K}$, some of the bluest objects showing similar colours to those presented by Mira variables. More evolved objects are usually associated to the reddest colours while there seems to exist a clear correlation between the near infrared colours and the phase, the reddest colours appearing associated to the minimum brightness.
\end{abstract}

It is our intention to study the photometric properties observed in the near infrared and the correlation of the amplitudes and periods found with their characteristics derived from IRAS data (colour, variability index,...) and from $\mathrm{OH}$ maser measurements (expansion velocity, luminosity,...). A substantial progress will be made when more data will be available. This will help us to better understand the short transition phase after the end of the AGB phase which precedes to the formation of a new planetary nebula $(\mathrm{PN})$, poorly known because of the lack of observational data. 